\title{
Erratum to: Oxcarbazepine monotherapy in patients with brain tumor-related epilepsy: open-label pilot study for assessing the efficacy, tolerability and impact on quality of life
}

\author{
M. Maschio $\cdot$ L. Dinapoli $\cdot$ F. Sperati $\cdot$ \\ A. Fabi $\cdot$ A. Pace $\cdot$ A. Vidiri $\cdot$ P. Muti
}

Published online: 5 October 2011

(C) Springer Science+Business Media, LLC. 2011

Erratum to: J Neurooncol

DOI 10.1007/s11060-011-0689-z

An important table was missing in the original publication. It was the table referred to in the Results section (in the first paragraph as the 'table in the supplemental materials' and in the subsection 'Tolerability' as 'Table'). The table is shown in this erratum.

The online version of the original article can be found under doi:10.1007/s11060-011-0689-z.

M. Maschio $(\square) \cdot$ L. Dinapoli

Department of Neuroscience and Cervical-Facial Pathology,

Center for Tumor-related Epilepsy, National Institute for Cancer,

"Regina Elena", Via Elio Chianesi 53, 00144 Rome, Italy

e-mail: maschio@ifo.it

M. Maschio - L. Dinapoli · A. Pace

Department of Neuroscience and Cervical-Facial Pathology,

Neurology Unit, National Institute for Cancer, "Regina Elena",

Via Elio Chianesi 53, 00144 Rome, Italy

F. Sperati

Department of Epidemiology, National Institute for Cancer,

"Regina Elena", Via Elio Chianesi 53, 00144 Rome, Italy

A. Fabi

Department of Oncology, National Institute for Cancer, "Regina

Elena", Via Elio Chianesi 53, 00144 Rome, Italy

\section{A. Vidiri}

Department of Radiology, National Institute for Cancer, "Regina

Elena”, Via Elio Chianesi 53, 00144 Rome, Italy

P. Muti

Scientific Direction, National Institute for Cancer, "Regina

Elena”, Via Elio Chianesi 53, 00144 Rome, Italy 


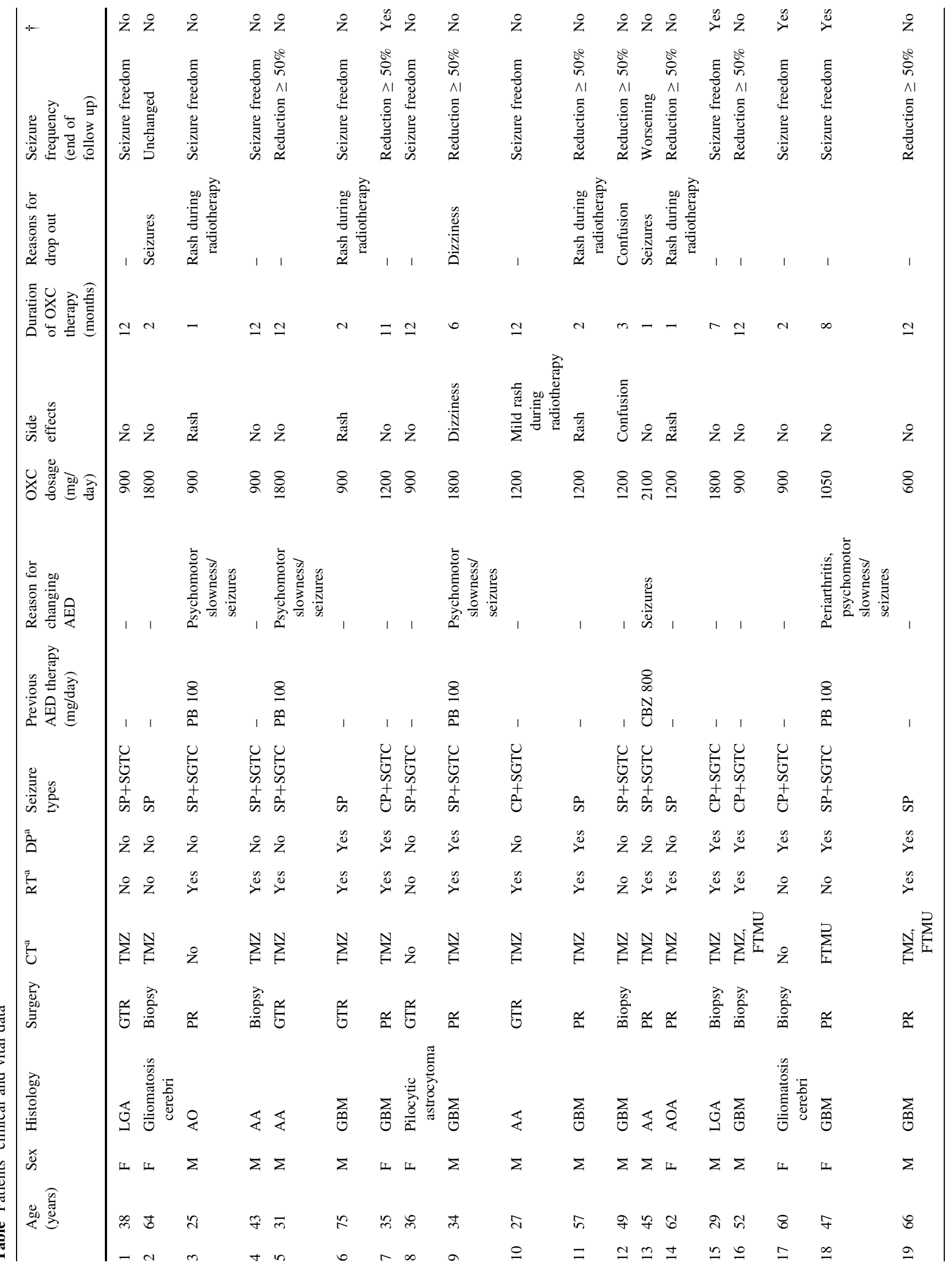




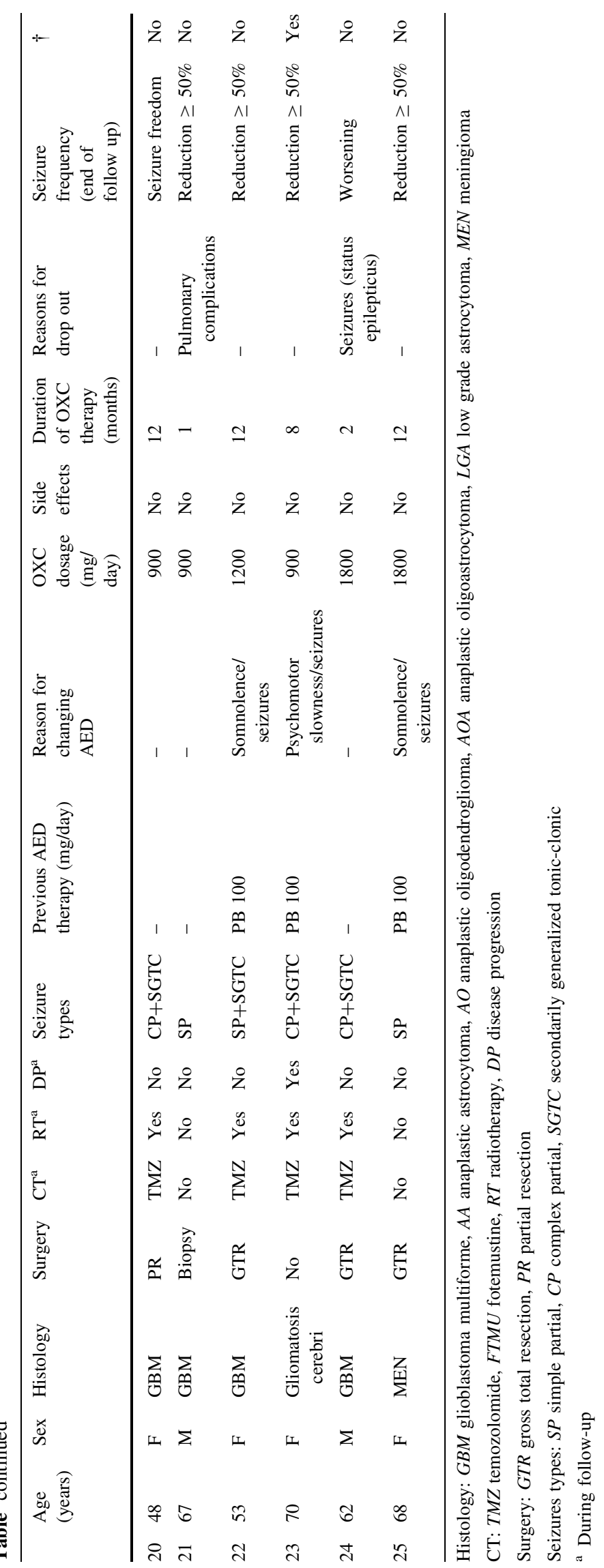

